\title{
A Finite-Volume Particle Method for Compressible Flows
}

\author{
D. Hietel ${ }^{1}$ K. Steiner ${ }^{1}$ and J. Struckmeier ${ }^{2}$
}

\begin{abstract}
We derive a new class of particle methods for conservation laws, which are based on numerical flux functions to model the interactions between moving particles. The derivation is similar to that of classical Finite-Volume methods; except that the fixed grid structure in the Finite-Volume method is substituted by so-called mass packets of particles. We give some numerical results on a shock wave solution for Burgers equation as well as the well-known one-dimensional shock tube problem.
\end{abstract}

\section{Introduction}

The Smoothed Particle Hydrodynamics method (SPH) as discussed by Monaghan in Ref. [5] is a quite popular particle scheme for problems governed by conservation laws, like problems in astrophysics, magneto-hydrodynamics or dense gas simulations. The basic idea behind the SPH method is to approximate the conservative quantities by a finite set of Lagrangian particles, which interact via molecular forces obtained from a smoothing procedure of the underlying conservation law.

A generic feature of conservation laws is that the solution will develop discontinuities as well as shock waves, even if the initial data are smooth functions. In order to handle this kind of weak solutions numerically applying the SPH method, one needs to introduce an artificial viscosity in the SPH formulation. This artificial viscosity is derived from a heuristic molecular model for interacting particles and, in particular, contains some fit parameters, which need to be chosen on the basis of numerical experiments.

In classical computational fluid dynamics, the Finite-Volume method is a common simulation scheme for compressible Euler equations and other systems of conservation laws [3]. The main feature in this scheme is, that the discrete quantities are obtained by averaging the conservative (or even the primitive) quantities over some spatial and fixed cells and the averaged flux function is approximated by a so-called numerical flux function, which a-priori includes some numerical viscosity.

Applying a Finite-Volume scheme requires the generation of a spatial grid, which may be structured or unstructured, and the grid generation becomes quite difficult, if the underlying geometry of the problem is complicated. Moreover, if one considers fluid dynamic

\footnotetext{
${ }^{1}$ ITWM, Erwin-Schrödinger Str., 67663 Kaiserslautern, Germany

${ }^{2}$ Dept. of Mathematics, University of Kaiserslautern, P.O Box 3049, 67653 Kaiserslautern, Germany, email: struckm@mathematik.uni-kl.de
} 
problems, which contain a fluid-structure interaction or some flexible structure within the flow, one needs to adapt the finite-volume grid in each time step, which again is a nontrivial procedure. Here, it may be more appropriate to use particle schemes based on Lagrangian particles, like the SPH method, because these schemes are formulated completely grid-free. But, one may even try to combine the generic features of a Finite-Volume scheme and a particle method, namely the concept of a numerical flux function and the flow description using Lagrangian particles and the aim of the present paper is to demonstrate, how one may derive a combined Finite-Volume particle scheme. In Section 2, we shortly recall the basic features of the Smoothed Particle Hydrodynamics method and discuss, how one includes an artificial viscosity in the equations of motion. A combined Finite-Volume particle scheme is derived in Section 3 and here we mainly focuse on the way, how to include a numerical flux function. Some numerical results for two one-dimensional test problems, namely a shock wave solution for the (inviscid) Burgers equation and the well-known shock tube problem governed by the (isentropic) Euler equations, are given in Section 4.

\section{The Smoothed Particle Hydrodynamics Method}

In the following we discuss a popular particle method for compressible Euler equations (mainly used in astrophysics), namely the Smoothed Particle Hydrodynamics method (SPH) as discussed by Monaghan in Ref. [5]. The basic idea behind the SPH method is, that each flow quantity $A(t, \mathbf{x})$, like the velocity or pressure field, is smoothed by a smoothing kernel $W^{\prime}\left(\mathbf{x}, \mathbf{x}_{*}\right)$ with respect to a measure associated to the mass density $\rho(t, \mathbf{x})$ of the flow,

$$
A_{s}(t, \mathbf{x})=\int_{\mathbb{R}^{3}} \frac{A\left(t, \mathbf{x}_{*}\right)}{\rho\left(t, \mathbf{x}_{*}\right)} W^{\prime}\left(\mathbf{x}, \mathbf{x}_{*}\right) \rho\left(t, \mathbf{x}_{*}\right) \mathrm{d} \mathbf{x}_{*}
$$

Then, the smoothed quantities are approximated by a set of Lagrangian particles, similar to a Monte Carlo integration of the integral term appearing in (2.1),

$$
A_{s}(t, \mathbf{x}) \approx \sum_{i=1}^{n} m_{i} \frac{A_{i}}{\rho_{i}} W^{\prime}\left(\mathbf{x}, \mathbf{x}_{i}\right)
$$

Here, the particles are located at the spatial positions $\mathbf{x}_{i}(t), i=1, \ldots, n$ and, as Lagrangian particles, they are moving along the trajectories $\dot{\mathbf{x}}_{i}=\mathbf{v}_{i}$, where $\mathbf{v}_{i}(t)$ denotes the velocity of the $i$-th particle.

The smoothing kernel $\widehat{W}\left(\mathbf{x}, \mathbf{x}_{*}\right)$ is in general radial symmetric, because there are no preferred directions in the smoothing procedure, i.e.

$$
\widehat{W}\left(\mathbf{x}, \mathbf{x}_{*}\right)=W_{h}\left(\left\|\mathbf{x}-\mathbf{x}_{*}\right\|\right)=\frac{1}{h^{d}} f\left(\frac{r}{h}\right)
$$

where $h$ denotes the so-called smoothing length, defining the decay of the kernel with increasing radial distance $r=\left\|\mathbf{x}-\mathbf{x}_{*}\right\|$. The function $f$ in (2.3) denotes a shape function ( $d$ is the spatial dimension) and from a theoretical point of view, the best choice for the shape function seems to be a Gaussian kernel. This kernel is reproduced by differentiation and the Fourier transform itself is again Gaussian, which simplifies the stability analysis of 
the SPH method. In practice, it is more appropriate to use kernels with compact support - otherwise one has to consider long-range interactions between single particles. For a detailed investigation on various SPH smoothing kernels we refer the reader to Ref. [2].

In the case of an isentropic flow, i.e. $P=P(\rho)$, the equations of motion in the SPH method are given by

$$
\begin{aligned}
\dot{\mathbf{x}}_{i} & =\mathbf{v}_{i} \\
\dot{\mathbf{v}}_{i} & =-\sum_{j=1}^{n} m_{j}\left(\frac{P_{i}}{\rho_{i}^{2}}+\frac{P_{j}}{\rho_{j}^{2}}\right) \nabla W_{i j}, \quad \nabla W_{i j}=\nabla_{x} \widehat{W}\left(\mathbf{x}_{i}, \mathbf{x}_{j}\right)
\end{aligned}
$$

where each particle carries the information $\left(m_{i}, \mathbf{x}_{i}(t), \mathbf{v}_{i}(t)\right)$. Moreover, the density field is obtained via the interpolation formula defined by (2.2), i.e.

$$
\rho_{i}=\sum_{i=1}^{n} m_{j} W_{i j}, \quad W_{i j}=\widehat{W}\left(\mathbf{x}_{i}, \mathbf{x}_{j}\right)
$$

The right hand side of $(2.5)$ defines the particle interactions by a force term $F_{i j}$

$$
F_{i j}=m_{i} m_{j}\left(\frac{P_{i}}{\rho_{i}^{2}}+\frac{P_{j}}{\rho_{j}^{2}}\right) \nabla W_{i j}
$$

between the $i$-th and $j$-th particle. This is the reason, why the Gaussian kernel is substituted by a smoothing kernel with compact support: to reduce the total number of particle interactions to the order $O(n)$.

Eq. (2.5) as well as (2.6) are not unique in SPH, in the sense that there exist some other representations for the equations of motion in SPH [5]. E.g., the calculation of a particle density $\rho_{i}$ by the interpolation rule $(2.6)$ is referred as "density by summation" and an alternative way to obtain the density of a single particle is the so-called "density by continuity equation": this approach uses an additional evolution equation of the form

$$
\frac{\mathrm{d} \rho_{i}}{\mathrm{~d} t}=\sum_{i=1}^{n} m_{j}\left(\mathbf{v}_{i}-\mathbf{v}_{j}\right) \nabla W_{i j}
$$

where $\rho_{i}$ denotes the density of the $i$-th particle. Formally, both methods are equal, if the initial condition for Eq. (2.7) is consistent with the interpolation rule, but due to numerical integration of (2.7), the results will be different using (2.7) instead of (2.6).

The equations of motion of the SPH method are numerically integrated by standard timeintegration schemes for ordinary differential equations. The most popular scheme - typically used in SPH applications - is the so-called modified Euler scheme (often referred as a predictor-corrector scheme). One can show, that the stability of the scheme is nearly identical to that of a more sophisticated Leapfrog or high-order Runge-Kutta scheme. The time integration yields a typical CFL-condition for explicit schemes applied to hyperbolic systems. This CFL-condition needs to be modified by the artificial viscosity term, which is introduced to handle shock solutions.

Like other numerical schemes for the compressible Euler/Navier-Stokes equations, the SPH method gets into problems in the low-Mach number limit. The equations of motions (2.4), 
(2.5) form a stiff system, because in the equations for the velocities of the SPH particles appears the inverse Mach number on the right hand side of the equation. Applying an explicit time integration yields extremely small time steps and there is still a need for better integration techniques (even some modified SPH formulations) in the low-Mach number limit.

In order to treat shocks with the SPH method, it is necessary to add an artificial viscosity term in the evolution equations for the particle velocities, i.e.

$$
\dot{\mathbf{v}}_{i}=-\sum_{j=1}^{n} m_{j}\left(\frac{P_{i}}{\rho_{i}^{2}}+\frac{P_{j}}{\rho_{j}^{2}}+\Pi_{i j}\right) \nabla W_{i j}
$$

where $\Pi_{i j}$ denotes the artificial viscosity. Numerical experiments showed, that the standard viscosity terms like the bulk or the von Neumann-Richtmyer viscosity are not appropriate viscosity terms to be used in the SPH method. Hence, Monaghan proposed a so-called viscous "pressure" term, which is based on a model for real molecular interactions,

$$
\Pi_{i j}= \begin{cases}\frac{1}{\rho_{i j}}\left(-\alpha h c_{i j} \mu_{i j}+\beta h^{2} \mu_{i j}^{2}\right) & : \text { if } \mu_{i j} \leq 0 \\ 0 & : \text { else }\end{cases}
$$

and

$$
\mu_{i j}=\frac{\left(\mathbf{v}_{i}-\mathbf{v}_{j}\right)\left(\mathbf{x}_{i}-\mathbf{x}_{j}\right)}{\left\|\mathbf{x}_{i}-\mathbf{x}_{j}\right\|^{2}+\varepsilon h^{2}}
$$

The additional term $\varepsilon h^{2}$ in the denominator prevents the term $\mu_{i j}$ to be singular as $\mathbf{x}_{j} \rightarrow \mathbf{x}_{i}$. Moreover, the artificial viscosity term (2.8) contains two more fit parameters $\alpha$ and $\beta$, which are determined (as well as the value for $\varepsilon$ ) by numerical experiments, e.g., $(\alpha, \beta)=(1,2)$ and $\varepsilon=0.01$ for problems from gas dynamics (to treat high Mach number shocks) and $(\alpha, \beta)=(0.1,0)$ and $\varepsilon=0.01$ for problems from hydrodynamics.

The main advantage of the SPH method is the flow description by moving particles; in particular it is not necessary to introduce a spatial grid. The method is straightforward to implement and the equations of motion are given by an $n$-particle system with binary interactions, such that one has to integrate a system of ordinary differential equations to solve the equations of motion. Finally, the SPH scheme is quite flexible concerning different models; here, we refer the reader to the work by Monaghan, on multi-phase flows, dusty gas simulations or free surface flows. But, there are even some major drawbacks in the SPH scheme. The scheme is restricted to time-dependent and compressible flows, the accuracy is rather low and it contains some fluctuations, the implementation of boundary conditions is a bit tricky when dealing with curved boundaries. The heuristic derivation of an artificial viscosity term to stabilize the flow is not satisfactory; in particular due to the parameters, which have to be fitted by numerical experiments.

For flows close to the incompressible limit or low-Mach number flows, the system of ordinary differential equations forms a stiff system. Here, implicit schemes are appropriate, but costly: the Jacobian does not contain some appropriate structure, such that one applies a fixed point iteration instead of a Newton method. There exists only a few investigations on the convergence properties of the SPH method, like the one given by Oelschläger [8] and Vila [11], recently by Di Lisio, Grenier and Pulvirenti [1]. Hence, even some detailed 
investigations on error estimates in the SPH scheme do not exist: one may refer the SPH method to a Monte Carlo integration, such that the solution should converge with the order $O(1 / \sqrt{n})$, where $n$ denotes the number of particles [5]. Finally, it should be mentioned that some related particle schemes for compressible flows were recently published by Yserentant [12], [13], as well as some new results on the SPH method by Morris and Monaghan [7] and Morris et al. [6].

\section{Derivation of a Finite-Volume Particle Method}

We consider a system of conservation laws written in the form

$$
\partial_{t} \Phi+\nabla \cdot F(\Phi)=0
$$

where $\Phi$ denotes the vector of conservative variables, $\Phi=\Phi(t, \mathbf{x}), \Phi \in \mathbb{R}^{m}$ for $t \geq 0$ and $\mathbf{x} \in \Omega \subset \mathbb{R}^{3}$. In addition, $F(\Phi)$ defines the flux vector of the conservation laws.

Remark 3.1

We are in particular interested on the derivation of a mixed Finite-Volume particle method for compressible flows governed by the Euler equations. Then, the conservative variables are given by $\Phi=(\rho, \rho \mathbf{u}, \rho e)$, where $\rho$ denotes the density, $\mathbf{u}$ the velocity and $e$ the specific energy. Then, the flux vector is given by

$$
F(\Phi)=\left(\begin{array}{c}
\rho \mathbf{u} \\
\rho(\mathbf{u} \otimes \mathbf{u})+p \\
(\rho e+p) \mathbf{u}
\end{array}\right)
$$

where $p$ denotes the pressure.

We start the derivation of the new particle method with some basic notations used in the following. The conservative quantities defined by the vector $\Phi$ should be approximated at each time by a finite set of particles, which are located inside the spatial domain $\Omega$. A particle, located at time $t$ at the spatial position $\mathbf{x}_{i}(t)$, is smeared out using a so-called mass packet $W_{i}(t, \mathbf{x})$, where $W_{i}$ depends on the particle position $\mathbf{x}_{i}(t)$ and the mass packet is obtained from a generic shape function $W(\mathbf{x})$ via the relation

$$
W_{i}(t, \mathbf{x})=m_{i} W\left(\mathbf{x}-\mathbf{x}_{i}(t)\right),
$$

where $m_{i}$ denotes the mass of the particle.

Given a set of particles, located at the positions $\mathbf{x}_{i}(t), i=1, \ldots, n$, one may define a mass density $\sigma(t, \mathbf{x})$ by

$$
\sigma(t, \mathbf{x})=\sum_{i=1}^{n} W_{i}(t, \mathbf{x})
$$

The conservation laws (3.1) should be appoximated by particles; hence we need some other discrete particle characteristics $\Phi_{i}(t)$ for each particle and to derive corresponding equations of motion for the discrete quantities $\Phi_{i}(t)$.

In the classical Finite-Volume method the discrete quantities are obtained from averages over some spatial cell $\nu_{i}$ with a finite volume $V_{i}$, where the cells form a fixed grid structure 
and, formally, the equations of motion are obtained from a weak form of (3.1) by testing the conservation laws against characteristic functions $\mathbb{I}_{\nu_{i}}$ of the single cells $\nu_{i}$.

A similar approach is used in the following; but the system (3.1) should be approximated by particles, i.e. we need a different set of test functions, which are related with the particle positions $\mathbf{x}_{i}(t)$. This set of test functions is obtained using the mass packets $W_{i}(t, \mathbf{x})$ of the particles given above: in particular, we define test functions $\Psi_{i}(t, \mathbf{x})$ in the form

$$
\Psi_{i}(t, \mathbf{x})=\frac{W_{i}(t, \mathbf{x})}{\sigma(t, \mathbf{x})}
$$

where the mass density $\sigma(t, \mathbf{x})$ is defined by (3.2). These test functions possess similar properties like the characteristic functions $\mathbb{1}_{\nu_{i}}$ used in the standard Finite-Volume schemes, in particular they yield a partition of the unity, i.e.

$$
\sum_{i=1}^{n} \Psi_{i}(t, \mathbf{x})=1
$$

for all $t \geq 0$ and $\mathbf{x} \in \mathbb{R}^{3}$ and we obtain straightforward the relation

$$
\sum_{i=1}^{n} \nabla_{x} \Psi_{i}(t, \mathbf{x})=0
$$

Moreover, we may define for each particle a (time-dependent) "volume" $V_{i}(t)$ given by

$$
V_{i}(t)=\int \Psi_{i}(t, \mathbf{x}) \mathrm{d} \mathbf{x}
$$

Now we test the conservation laws (3.1) against the new set of test functions $\Psi_{i}(t, \mathbf{x})$, i.e. we consider the weak form given by

$$
\int\left(\partial_{t} \Phi+\nabla \cdot F(\Phi)\right) \Psi_{i}(t, \mathbf{x}) \mathrm{d} \mathbf{x}=0
$$

for all $i=1, \ldots, n$, which yields the equations

$$
\frac{\mathrm{d}}{\mathrm{d} t} \int \Psi_{i}(t, \mathbf{x}) \Phi(t, \mathbf{x}) \mathrm{d} \mathbf{x}=\int \partial_{t} \Psi_{i}(t, \mathbf{x}) \Phi(t, \mathbf{x}) \mathrm{d} \mathbf{x}+\int F(\Phi)(t, \mathbf{x}) \nabla \Psi_{i}(t, \mathbf{x}) \mathrm{d} \mathbf{x}
$$

\section{Remark 3.2}

We consider for a moment the system of conservation laws as a pure Cauchy problem, i.e. on the whole spatial domain $\Omega=\mathbb{R}^{3}$. Hence, there appear no boundary integrals in the formula given above. The situation changes, if $\Omega$ is a bounded domain in $\mathbb{R}^{3}$ and the boundary integrals need to be evaluated using the prescribed boundary conditions.

For the two terms on the right hand side of (3.6), one obtains the following expressions.

\section{Proposition 3.3}

With the notations given above, we have

$$
\partial_{t} \Psi_{i}(t, \mathbf{x})=\sum_{j=1}^{n}\left(\dot{\mathbf{x}}_{j}(t) \Psi_{i}(t, \mathbf{x}) \frac{\nabla W_{j}(t, \mathbf{x})}{\sigma(t, \mathbf{x})}-\dot{\mathbf{x}}_{i}(t) \Psi_{j}(t, \mathbf{x}) \frac{\nabla W_{i}(t, \mathbf{x})}{\sigma(t, \mathbf{x})}\right)
$$




\section{Proof}

We compute, using $\partial_{t} W_{i}=-\dot{\mathbf{x}}_{i} \nabla W_{i}$,

$$
\begin{aligned}
\partial_{t} \Psi_{i}(t, \mathbf{x}) & =-\dot{\mathbf{x}}_{i}(t) \frac{\nabla W_{i}(\mathbf{x})}{\sigma(t, \mathbf{x})}+\frac{W_{i}(\mathbf{x})}{\sigma(t, \mathbf{x})^{2}} \sum_{j=1}^{n} \dot{\mathbf{x}}_{j}(t) \nabla W_{j}(\mathbf{x}) \\
& =-\dot{\mathbf{x}}_{i}(t) \frac{\nabla W_{i}(\mathbf{x})}{\sigma(t, \mathbf{x})}+\frac{\Psi_{i}(t, \mathbf{x})}{\sigma(t, \mathbf{x})} \sum_{j=1}^{n} \dot{\mathbf{x}}_{j}(t) \nabla W_{j}(\mathbf{x}) \\
& =\sum_{j=1}^{n}\left(\dot{\mathbf{x}}_{j}(t) \Psi_{i}(t, \mathbf{x}) \frac{\nabla W_{j}(t, \mathbf{x})}{\sigma(t, \mathbf{x})}-\dot{\mathbf{x}}_{i}(t) \Psi_{j}(t, \mathbf{x}) \frac{\nabla W_{i}(t, \mathbf{x})}{\sigma(t, \mathbf{x})}\right)
\end{aligned}
$$

where in the last step we used the normalization condition (3.4).

\section{Proposition 3.4}

The term $\nabla \Psi_{i}(t, \mathbf{x})$ may be expressed in the symmetric form

$$
\nabla \Psi_{i}(t, \mathbf{x})=\sum_{j=1}^{n}\left(\Psi_{j}(t, \mathbf{x}) \frac{\nabla W_{i}(t, \mathbf{x})}{\sigma(t, \mathbf{x})}-\Psi_{i}(t, \mathbf{x}) \frac{\nabla W_{j}(t, \mathbf{x})}{\sigma(t, \mathbf{x})}\right)
$$

\section{Proof}

We compute first

$$
\nabla \Psi_{i}(t, \mathbf{x})=\frac{1}{\sigma(t, \mathbf{x})} \nabla W_{i}(t, \mathbf{x})-\frac{W_{i}(t, \mathbf{x})}{\sigma(t, \mathbf{x})^{2}} \sum_{j=1}^{n} \nabla W_{j}(t, \mathbf{x}),
$$

and applying (3.4) in the same way as above yields (3.8).

Using the two propositions given above, we obtain from the weak form of our conservation laws the expression

$$
\begin{aligned}
& \frac{\mathrm{d}}{\mathrm{d} t} \int \Phi(t, \mathbf{x}) \Psi_{i}(t, \mathbf{x}) \mathrm{d} \mathbf{x}=\sum_{j=1}^{n} \int F(\Phi)(t, \mathbf{x})\left(\Psi_{j}(t, \mathbf{x}) \frac{\nabla W_{i}(\mathbf{x})}{\sigma(t, \mathbf{x})}-\Psi_{i}(t, \mathbf{x}) \frac{\nabla W_{j}(\mathbf{x})}{\sigma(t, \mathbf{x})}\right) \mathrm{d} \mathbf{x} \\
& +\sum_{j=1}^{n}\left(\int \Phi(t, \mathbf{x})\left(\dot{\mathbf{x}}_{j}(t) \Psi_{i}(t, \mathbf{x}) \frac{\nabla W_{j}(\mathbf{x})}{\sigma(t, \mathbf{x})}-\dot{\mathbf{x}}_{i}(t) \Psi_{j}(t, \mathbf{x}) \frac{\nabla W_{i}(\mathbf{x})}{\sigma(t, \mathbf{x})}\right) \mathrm{d} \mathbf{x}\right)
\end{aligned}
$$

The term on the left hand side of the equation above yields an appropriate expression to define discrete and averaged quantities, which may be related to particle characteristics. Hence, we define together with the volume $V_{i}(t)$ of a particle the discrete characteristics $\Phi_{i}(t)$ via the relation

$$
\Phi_{i}(t)=\frac{1}{V_{i}} \int \Phi(t, \mathbf{x}) \Psi_{i}(t, \mathbf{x}) \mathrm{d} \mathbf{x}
$$

and the term on the left hand side of (3.9) is then substituted by the expression $\frac{\mathrm{d}\left(V_{i} \Phi_{i}\right)}{\mathrm{d} t}$. In the next step, we have to explain how to evaluate the two integrals on the right hand side of (3.9). For the second term we use the approximation

$$
\begin{aligned}
\int \Phi(t, x) \Psi_{i}(t, \mathbf{x}) \frac{\nabla W_{j}}{\sigma(t, \mathbf{x})} \mathrm{d} \mathbf{x} & \approx \frac{1}{V_{i}} \int \Phi \Psi_{i}(t, \mathbf{x}) \mathrm{d} \mathbf{x} \cdot\left(\int \frac{W_{i}(t, \mathbf{x}) \nabla W_{j}(t, \mathbf{x})}{\sigma^{2}(t, \mathbf{x})} \mathrm{d} \mathbf{x}\right) \\
& =\gamma_{i j} \Phi_{i}
\end{aligned}
$$


where the (time-dependent) coefficients $\gamma_{i j}$ are given by

$$
\gamma_{i j}(t)=\int \frac{W_{i}(t, \mathbf{x}) \nabla W_{j}(t, \mathbf{x})}{\sigma^{2}(t, \mathbf{x})} \mathrm{d} \mathbf{x}
$$

We use this kind of approximation, because the discrete quantities of our particles are given by (3.10) and we divide the integral on the left hand side of (3.11) into two integrals, where the first one yields exactly these quantities.

Hence, the complete expression reads

$$
\sum_{j=1}^{n} \int \Phi\left(\dot{\mathbf{x}}_{j} \Psi_{i} \frac{\nabla W_{j}}{\sigma}-\dot{\mathbf{x}}_{i} \Psi_{j} \frac{\nabla W_{i}}{\sigma}\right) \mathrm{d} \mathbf{x}=\sum_{j=1}^{n}\left(\Phi_{i} \dot{\mathbf{x}}_{j} \gamma_{i j}-\Phi_{j} \dot{\mathbf{x}}_{i} \gamma_{j i}\right)
$$

Concerning the first term, we use an approximation, which corresponds to a Finite-Volume method, i.e. we apply the approximation

$$
\sum_{j=1}^{n} \int F(\Phi)\left(\Psi_{j} \frac{\nabla W_{i}}{\sigma}-\Psi_{i} \frac{\nabla W_{j}}{\sigma}\right) \mathrm{d} \mathbf{x} \approx-\sum_{j=1}^{n} \beta_{i j}(t) g\left(\Phi_{i}, \Phi_{j}, n_{i j}\right)
$$

where the coefficients $\beta_{i j}$ are given by $\beta_{i j}=\gamma_{i j}-\gamma_{j i}$ and the normal vector $n_{i j}$ is obtained from the equation

$$
n_{i j}=\frac{\beta_{i j}}{\left\|\beta_{i j}\right\|}
$$

Moreover, the function $g(\cdot, \cdot, \cdot)$ is related with a numerical flux function as used in standard Finite-Volume schemes, e.g., we propose the consistency relation $g(\Phi, \Phi, n)=F(\Phi)$.

Together with the two approximations (3.12) and (3.13), we obtain the equations of motion for our new particle scheme in the form

$$
\frac{\mathrm{d}}{\mathrm{d} t}\left(V_{i} \Phi_{i}\right)=-\sum_{j=1}^{n} \beta_{i j} g\left(\Phi_{i}, \Phi_{j}, n_{i j}\right)+\sum_{j=1}^{n}\left(\gamma_{i j} \dot{\mathbf{x}}_{j} \Phi_{i}-\gamma_{j i} \dot{\mathbf{x}}_{i} \Phi_{j}\right)
$$

together with the (time-dependent) coefficients $\gamma_{i j}$ and $\beta_{i j}$ as defined above. On the other hand, we have still the freedom to choose, how particles should move, because the terms $\dot{\mathbf{x}}_{i}$ are not specified up to now and obviously there are two generic choices for the particle velocities: the first one, from which we recover the standard Finite-Volume method, is to use spatially fixed positions for the particles, i.e. $\dot{\mathbf{x}}_{i}=0$ for all $t \geq 0$ and $i=1, \ldots, n$. Then, as a consequence, we obtain that even the volumes of the particles are time-independent, i.e. $\dot{V}_{i}=0$ and the particle positions may be interpreted as the cell centers of a grid structure used in Finite-Volume methods.

The second generic choice - the one, we are mainly interested in - is to choose Lagrangian particles to approximate our conservation laws, i.e. we define $\dot{\mathbf{x}}_{i}=\mathbf{u}_{i}$, where $\mathbf{u}_{i}$ denotes the velocity of the $i$-th particle. Then even the volumes of the particles will change, because particles are moving and the equations of motion as given in (3.14) are not closed: on the left hand side appears the product $V_{i} \Phi_{i}$, whereas the right hand side only contains the discrete conservative quantities $\Phi_{i}$. Hence, in order to close the equations of motion, we 
need an additional equation for the volumes of particles. Here, one should remember the definition of the particle volumes in the form

$$
V_{i}(t)=\int \Psi_{i} \mathrm{~d} \mathbf{x}
$$

This equation might even be interpreted - dividing the equation by $V_{i}$ - as an integral average of the unity, similar to the definition of the discrete quantities $\Phi_{i}$. Hence, to obtain an equation for the volumes, it is appropriate to add the trivial equation

$$
\frac{\partial \mu(t, \mathbf{x})}{\partial t}=0
$$

where $\mu(t, \mathbf{x})=1$, for all $t \geq 0$ and $\mathbf{x} \in \mathbb{R}^{3}$ to the system of conservation laws. Then, the equations for the volumes read

$$
\frac{\mathrm{d} V_{i}}{\mathrm{~d} t}=\sum_{j=1}^{n}\left(\gamma_{i j} \mathbf{u}_{j}-\gamma_{j i} \mathbf{u}_{i}\right)
$$

A basic property of numerical schemes for conservation laws is the conservation property, which should hold on the discrete level applying a discrete approximation for the exact solution. This property is a-priori included in a standard Finite-Volume formulation, but even incorporated in the particle scheme presented above.

\section{Lemma 3.5}

The Finite-Volume particle method defined by the equations of motion (3.14) fulfills the discrete conservation property

$$
\frac{\mathrm{d}}{\mathrm{d} t}\left(\sum_{i}\left(V_{i} \Phi_{i}\right)\right)=0
$$

if the numerical flux function $g$ fulfills the symmetry condition

$$
g\left(\Phi_{i}, \Phi_{j}, n_{i j}\right)=g\left(\Phi_{j}, \Phi_{i}, n_{j i}\right)
$$

\section{Proof}

From the equations of motion (3.14) it follows, together with the symmetry condition (3.15)

$$
\begin{aligned}
\frac{\mathrm{d}}{\mathrm{d} t}\left(\sum_{i}\left(V_{i} \Phi_{i}\right)\right) & =\sum_{i} \frac{\mathrm{d}}{\mathrm{d} t}\left(V_{i} \Phi_{i}\right) \\
& =-\sum_{i, j}\left(\beta_{i j} g\left(\Phi_{i}, \Phi_{j}, n_{i j}\right)+\gamma_{j i} \dot{x}_{i} \Phi_{j}-\gamma_{i j} \dot{x}_{j} \Phi_{i}\right) \\
& =\sum_{i, j}\left(\gamma_{j i}\left(g\left(\Phi_{j}, \Phi_{i}, n_{j i}\right)+\dot{x}_{i} \Phi_{j}\right)-\gamma_{i j}\left(g\left(\Phi_{i}, \Phi_{j}, n_{i j}\right)+\dot{x}_{j} \Phi_{i}\right)\right) \\
& =0
\end{aligned}
$$




\section{Example 3.6}

As a first example we consider the well-known Burgers equation

$$
u_{t}+f(u)_{x}=0, \quad f(u)=u^{2} / 2
$$

Besides the spatial position $x_{i}(t)$, each particle carries the additional quantities $u_{i}(t)$ and the particle volume $V_{i}(t)$. The equations of motion read

$$
\begin{aligned}
\frac{\mathrm{d} x_{i}}{\mathrm{~d} t} & =\frac{u_{i}}{2} \\
\frac{\mathrm{d} u_{i}}{\mathrm{~d} t} & =-\sum_{i=1}^{n} \beta_{i j}\left(\frac{u_{i} u_{j}}{2}-g_{i j}\right) \\
\frac{\mathrm{d} V_{i}}{\mathrm{~d} t} & =\frac{1}{2} \sum_{i=1}^{n}\left(\gamma_{i j} u_{j}-\gamma_{j i} u_{i}\right)
\end{aligned}
$$

In Section 4, we discuss the Burgers equation in more detail and give some numerical results using an upwind scheme for the numerical flux function $g_{i j}$. Moreover, the numerical solution $u(t, x)$ is obtained via the interpolation formula

$$
u(t, x)=\frac{1}{\sigma(t, x)} \sum_{i=1}^{n} u_{i} W_{i}(x)
$$

and

$$
\sigma(t, x)=\sum_{i=1}^{n} W_{i}(x)
$$

\section{Numerical Examples}

In the following we give some numerical results applying the Finite-Volume particle method derived in the previous section and compare the results with a classical Finite-Volume scheme. We restrict ourselve to simple one-dimensional problems, because we are mainly interested in investigating the general possibility to use a numerical flux function in combination with moving particles. As smoothing kernel for the Finite-Volume particle method we use a third order polynomial truncated at a smoothing length $h$ defined by

$$
W(x ; h)= \begin{cases}2 \frac{|x| x^{2}}{h^{4}}-3 \frac{x^{2}}{h^{3}}+\frac{1}{h} & :|x| \leq h \\ 0 & :|x|>h\end{cases}
$$

with derivative

$$
W^{\prime}(x ; h)= \begin{cases}6 \frac{x|x|}{h^{4}}-6 \frac{x}{h^{3}} & :|x| \leq h \\ 0 & :|x|>h\end{cases}
$$

The kernel is chosen to fulfill the conditions

$$
\begin{aligned}
\int_{[-h, h]} W(x ; h) \mathrm{d} x & =1 \\
W(h ; h) & =W(-h ; h)=0 \\
W^{\prime}(h ; h) & =W^{\prime}(-h ; h)=0
\end{aligned}
$$


Moreover, we have the symmetry $W^{\prime}(-x ; h)=-W^{\prime}(x ; h)$ and therefore

$$
\int_{[-h, h]} W^{\prime}(x ; h) \mathrm{d} x=0
$$

\subsection{The Burgers Equation}

In this section we give numerical results obtained for a Riemann problem of the Burgers equation

$$
u_{t}+f(u)_{x}=0, \quad f(u)=\frac{u^{2}}{2}
$$

with initial data

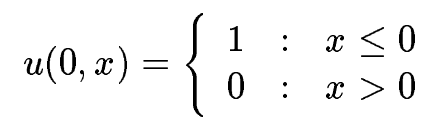

and compare the Finite-Volume particle method with a classical Finite-Volume scheme. A standard Finite-Volume method may be written in the form

$$
u_{j}^{n+1}=u_{j}^{n}-\frac{\Delta t}{\Delta x}\left(g\left(u_{j}^{n}, u_{j+1}^{n}\right)-g\left(u_{j-1}^{n}, u_{j}^{n}\right)\right)
$$

where $g$ denotes the numerical flux function, $\Delta x$, respectively, $\Delta t$ the space and time discretization of the Finite-Volume method and discrete quantities $u_{j}^{n}=u(n \Delta t, j \Delta x)$. In particular, we use an upwind scheme for the numerical flux function, such that $g(v, w)=$ $f(v)$, because the solution of the Riemann problem and therefore the characteristic speed is positive. The corresponding discrete equations applying a Finite-Volume particle method and an explicit, first order time integration are given by

$$
\begin{cases}x_{i}^{n+1} & =x_{i}^{n}+\Delta t f\left(u_{i}^{n}\right) \\ V_{i}^{n+1} & =V_{i}^{n}+\Delta t \sum_{j=1}^{n}\left(\gamma_{i j} f\left(u_{j}^{n}\right)-\gamma_{j i} f\left(u_{i}^{n}\right)\right) \\ (V u)_{i}^{n+1} & =(V u)_{i}^{n}-\Delta t \sum_{j=1}^{n}\left(\beta_{i j} \bar{g}\left(u_{i}^{n}, u_{j}^{n}\right)-\gamma_{i j} f\left(u_{j}^{n}\right) u_{i}^{n}+\gamma_{j i} f\left(u_{i}^{n}\right) u_{j}^{n}\right)\end{cases}
$$

where the numerical flux function $\bar{g}$ is given by

$$
\bar{g}\left(u_{i}, u_{j}\right)=\left\{\begin{array}{l}
f\left(u_{i}\right) \quad: \quad x_{i} \leq x_{j} \\
f\left(u_{j}\right) \quad: \quad x_{i}>x_{j}
\end{array}\right.
$$

Hence, each particle carries besides its spatial position the discrete quantities $(V u)_{i}$ and $V_{i}$, where the latter one defines the volume of a particle, $(V u)_{i}$ the aritificial quantity "Volume - Velocity" and we need to evaluate the numerical flux function $\bar{g}$ using the ratios $(V u)_{i} / V_{i}$, $i=1, \ldots, n$. Moreover, we consider Lagrangian particles, i.e. the particles are moving with velocity $(V u)_{i} /\left(2 V_{i}\right)$.

The simulations are performed on the spatial interval $[-5: 5]$ using 100 discrete points, either based on a equidistant grid, when applying the Finite-Volume method, or 100 particles, when applying the Finite-Volume particle method, where at initial time, the particles are located at the mid points of the corresponding Finite-Volume grid. The smoothing 
length $h$ is given by $h=1.8 \Delta x$, where $\Delta x$ denotes the length of a spatial cell in the FiniteVolume scheme. The time step $\Delta t$ is equal to $\Delta t=0.05$ and, as mentioned above, we use a simple explicit, first order time integration. Moreover, the geometry coefficient $\gamma_{i j}$ and $\gamma_{j i}$ are obtained via numerical integration.

In Fig. 4.1, we compare the numerical results obtained from the Finite-Volume method (4.2) and the Finite-Volume particle scheme (4.3) after 20 time steps, i.e. at time $t=1$, the corresponding results at time $t=4$ are given in Fig. 4.2.

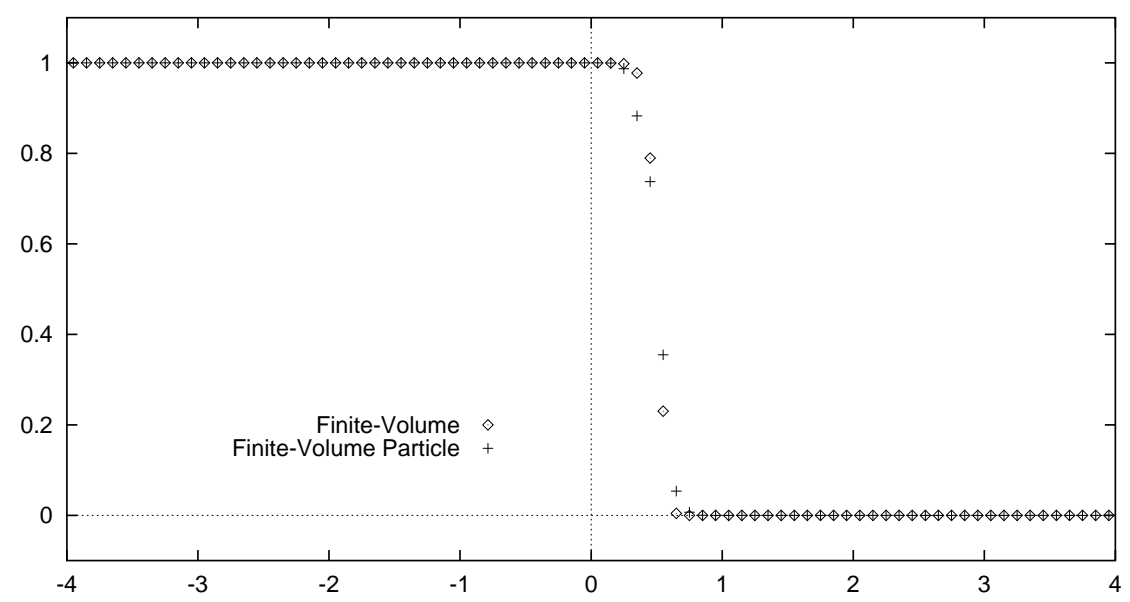

Fig. 4.1 Numerical Solutions for Burgers Equation at $t=1$

The numerical results indicate, that the concept of a numerical flux function implemented in a particle scheme yields an appropriate smoothing of the shock wave, which is slightly larger compared to a classical Finite-Volume scheme, due to the smoothing length $h=1.8 \Delta x$. On the other hand, it is not necessary to include an artificial viscosity term in the equations of motions, because the numerical flux function itself yields a sufficiently large numerical viscosity to surpress oscillations.

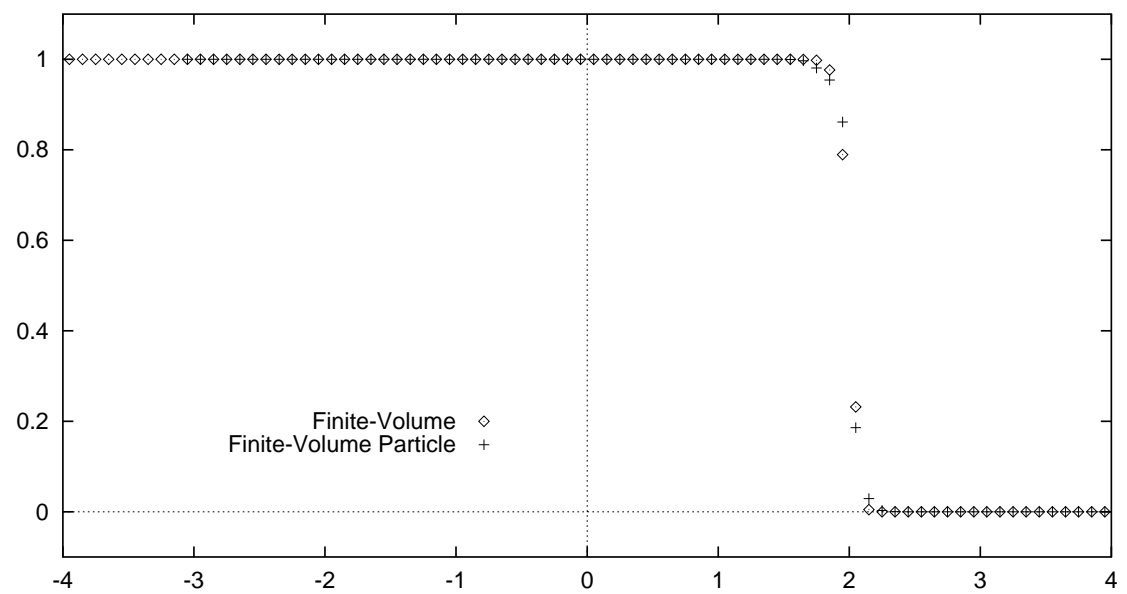

Fig. 4.2 Numerical Solutions for Burgers Equation at $t=4$

A further advantage of the new Finite-Volume particle approach compared to the standard $\mathrm{SPH}$ scheme is, that the numerical results turn out to be quite robust when changing the 
smoothing length $h$ of the smoothing kernel. Fig. 4.3 shows the numerical results obtained for two different values for the smoothing length, namely $h=1.2 \Delta x$, i.e. a smaller value compared to the results given in Fig. 4.2 , and $h=2.4 \Delta x$, i.e. a larger value compared to the previous choice $h=1.8 \Delta x$.

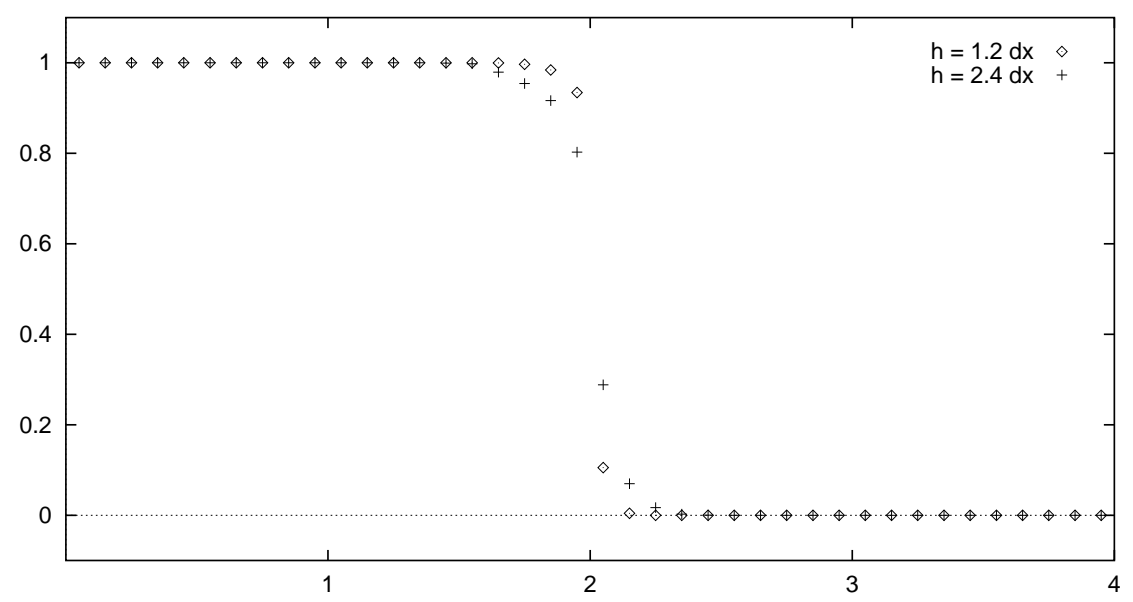

Fig. 4.3 Influence on the Smoothing Length $h$

Decreasing the smoothing length yields, as expected, a better resolution of the shock wave, without producing any kind of oscillations.

\subsection{Shock Tube Problem}

Here, we consider the simplified shock tube problem by restricting to the one-dimensional isentropic Euler equations given by

$$
U_{t}+F(U)_{x}=0
$$

with $U=\left(u_{1}, u_{2}\right)$, flux function $F(U)=\left(u_{2}, u_{2}^{2} / u_{1}+P\left(u_{1}\right)\right)^{t}$, pressure $P=c u_{1}^{\gamma}, c>0$ and initial conditions

$$
u_{1}(x)= \begin{cases}u_{1}^{l} & : x \leq 0 \\ u_{1}^{r} & : x>0\end{cases}
$$

and $u_{2}(x)=0$ for $x \in \mathbb{R}$. Moreover, to obtain a homogeneous flux, i.e. $F(\alpha U)=\alpha F(U)$, $\alpha \in \mathbb{R}$, we further restrict to the case $\gamma=1$. As numerical flux function we use a flux vector splitting scheme based on Steger and Warming, which yields

$$
g(U, V)=A^{+}(U) U+A^{-}(V) V
$$

where the matrices $A^{+}$and $A^{-}$are defined by

$$
A^{ \pm}=T \Lambda^{ \pm} T^{-1}
$$


with $\Lambda^{ \pm}=\operatorname{diag}\left(\lambda_{1}^{ \pm}, \lambda_{2}^{ \pm}\right), \lambda_{1}, \lambda_{2}$ the eigenvalues of $(D F)(U), T$ the matrix of right eigenvectors and $\lambda_{i}^{+}=\max \left\{\lambda_{i}, 0\right\}, \lambda_{i}^{-}=\min \left\{\lambda_{i}, 0\right\}$. In particular, one obtains the expressions

$$
A^{+}(U) U=\left\{\begin{array}{cl}
\left(\begin{array}{c}
u_{2} \\
u_{2}^{2} / u_{1}+P\left(u_{1}\right)
\end{array}\right) & : \lambda_{1}, \lambda_{2}>0 \\
0 & : \lambda_{1}, \lambda_{2}<0 \\
\frac{1}{2}\left(\begin{array}{c}
u_{2}+P\left(u_{1}\right) \\
(u+a)^{2} u_{1}
\end{array}\right) & : \lambda_{1}<0, \lambda_{2}>0 \\
\frac{1}{2}\left(\begin{array}{c}
u_{2}-P\left(u_{1}\right) \\
(u-a)^{2} u_{1}
\end{array}\right) & : \lambda_{1}>0, \lambda_{2}<0
\end{array}\right.
$$

with $\lambda_{1}=u-a, \lambda_{2}=u+a$ and $u=u_{2} / u_{1}, a^{2}=P / u_{1}$ as well as

$$
A^{-}(V) V=\left\{\begin{array}{cl}
0 & : \lambda_{1}, \lambda_{2}>0 \\
\left(\begin{array}{cl}
v_{2} \\
v_{2}^{2} / v_{1}+P\left(v_{1}\right)
\end{array}\right) & : \lambda_{1}, \lambda_{2}<0 \\
\frac{1}{2}\left(\begin{array}{c}
v_{2}-P\left(v_{1}\right) \\
(v-a)^{2} v_{1}
\end{array}\right) & : \lambda_{1}<0, \lambda_{2}>0 \\
\frac{1}{2}\left(\begin{array}{c}
v_{2}+P\left(v_{1}\right) \\
(v+a)^{2} v_{1}
\end{array}\right) & : \lambda_{1}>0, \lambda_{2}<0
\end{array}\right.
$$

with $\lambda_{1}=v-a, \lambda_{2}=v+a$ and $v=v_{2} / v_{1}, a^{2}=P / v_{1}$.

Applying a Finite-Volume scheme for the shock tube problem yields the discrete equations

$$
U_{j}^{n+1}=U_{j}^{n}-\frac{\Delta t}{\Delta x}\left(g\left(U_{j}^{n}, U_{j+1}^{n}\right)-g\left(U_{j-1}^{n}, U_{j}^{n}\right)\right)
$$

For the Finite-Volume particle method using an explicit first order time integration, we have the equations of motion

$$
\left\{\begin{array}{llc}
x_{i}^{n+1} & = & x_{i}^{n}+\Delta t f\left(U_{i}^{n}, V_{i}^{n}\right) \\
V_{i}^{n+1} & = & V_{i}^{n}+\Delta t \sum_{j=1}^{n}\left(\gamma_{i j} f\left(U_{j}^{n}, V_{j}^{n}\right)-\gamma_{j i} f\left(U_{i}^{n}, V_{i}^{n}\right)\right) \\
(V U)_{i}^{n+1}= & (V U)_{i}^{n}-\Delta t \sum_{j=1}^{n}\left(\beta_{i j} \bar{g}\left(U_{i}^{n}, U_{j}^{n}\right)-\right. \\
& \left.\gamma_{i j} f\left(U_{j}^{n}, V_{j}^{n}\right) U_{i}^{n}+\gamma_{j i} f\left(U_{i}^{n}, V_{i}^{n}\right) U_{j}^{n}\right)
\end{array}\right.
$$

where the function $f\left(U_{i}\right)$ defines, how the particles should move in time and the numerical flux $\bar{g}$ is given by

$$
\bar{g}\left(U_{i}, U_{j}\right)= \begin{cases}g\left(U_{i}, U_{j}\right) & x_{i}<x_{j} \\ g\left(U_{j}, U_{i}\right) & x_{i} \geq x_{j}\end{cases}
$$

\subsubsection{The Case $u_{1}^{l}=1.1$ and $u_{1}^{r}=1$}

In the first simulation, we consider a small variation in the density between the two initial states, separated by a membrane in the tube. In particular, we use a density of 1.1 in the 
left part and a density of 1.0 in the right part of the shock tube. Hence, one expects a moderate density shock wave moving to the right and a moderate rarefaction wave moving to the left, together with a density plateau separating these two states.

The simulations are performed on the spatial interval $[-10: 10]$ and we again use 100 points to approximate the exact solution. Moreover, we use the same parameter like in the previous section, i.e. the smoothing length $h$ is given by $h=1.8 \Delta x$ and the time step is given by $\Delta t=0.05$. Fig. 4.4 and 4.5 , respectively, show the density and velocity profiles at time $t=4$, i.e. after 80 time steps, applying the Finite-Volume scheme (4.4) and the Finite-Volume particle method (4.5), where the particles are moved with velocity $f\left(U_{i}, V_{i}\right)=\left(V u_{2}\right)_{i} /\left(V u_{1}\right)_{i}$, i.e. the term $f\left(U_{i}, V_{i}\right)$ exactly denotes the (discrete) velocity of the $i$-th particle, and therefore corresponds to the idea of Lagrangian particles.
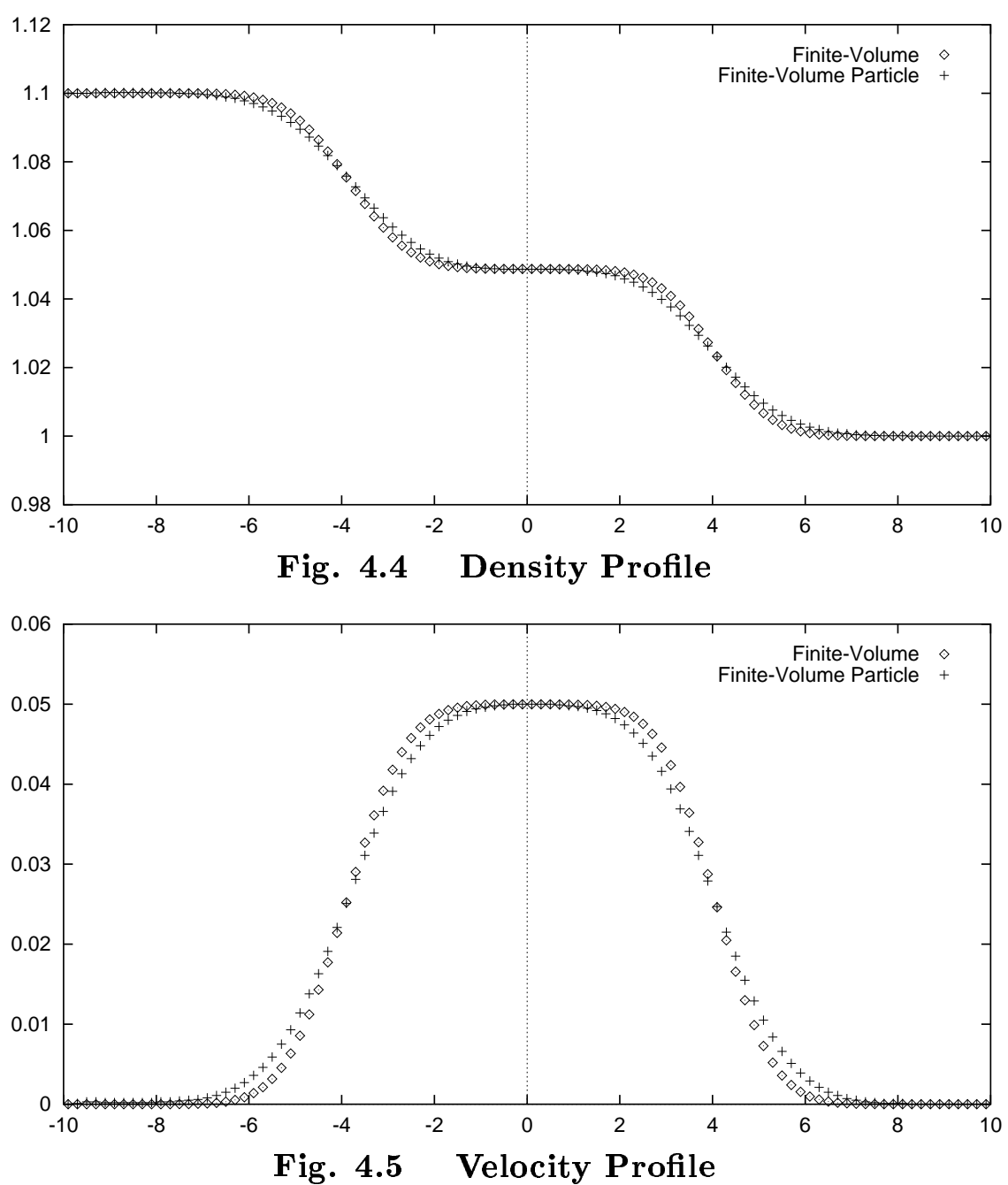

The discrete particle characteristics $(V U)_{i}$ and $V_{i}$ together with the particle positions $x_{i}$ at time $t=4$ are shown in Fig. 4.6. The results show, that the particle volumes as well as the products of the discrete density and the volume remain nearly constant at the initial value $20 / \Delta x$ and the same holds for the particle momentum, which remains close to zero. 


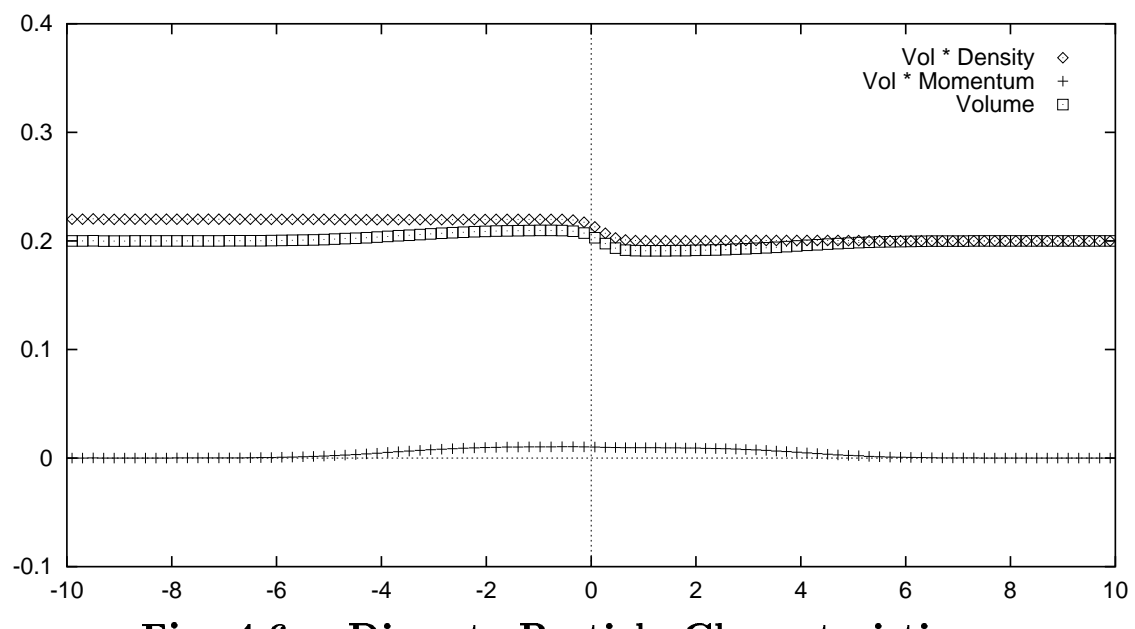

Fig. 4.6 Discrete Particle Characteristics

\subsubsection{The Case $u_{1}^{l}=1$ and $u_{1}^{r}=0.125$}

For the second configuration we increase the density variation between the left and right part of the shock problem to $u_{1}^{l} / u_{1}^{r}=8$ using the values $u_{1}^{l}=1$ and $u_{1}^{r}=0.125$. Hence, one may expect the formation of a strong density shock wave moving to the right together with a strong rarefaction wave moving to the left. Moreover, in contrast to the previous case, the flow will develop a non-vanishing velocity profile within the shock tube. The simulations use the numerical parameters as in Section 4.2.1, i.e. we compute the numerical solution on the interval $[-10: 10]$ using 100 discrete points.

In contrast to the previous example, where we considered a "weak" shock tube problem, the particles are moved according to the discrete velocity $f\left(U_{i}, V_{i}\right)=\left(V u_{2}\right)_{i} /\left(V u_{1}\right)_{i}$, which corresponds to the (discrete) momentum of a particle: the velocity profile within the shock tube will be of the order $O(1)$, such that in the rarefaction region the distance between two neighbouring particles will increase significantly and one should consider a variable smoothing length (or some other kind of adaptivity), when applying Lagrangian particles. This effect is reduced in the current simulation using the artificial velocity $\left(V u_{2}\right)_{i} /\left(V u_{1}\right)_{i}$, i.e. the momentum of a particle, which still fits into the derivation of the Finite-Volume particle scheme given by (3.14). Moreover, one may expect, due to the strong discontinuity in the initial condition, that the evaluation of the ratio of two computed quantities, which is necessary to determine the velocity of Lagrangian particles, may lead to numerical errors significantly larger than the original ones.

The resulting density and velocity profile are presented in Fig. 4.7 and 4.8, respectively, again we show the results after 80 time steps, i.e. at time $t=4$. 


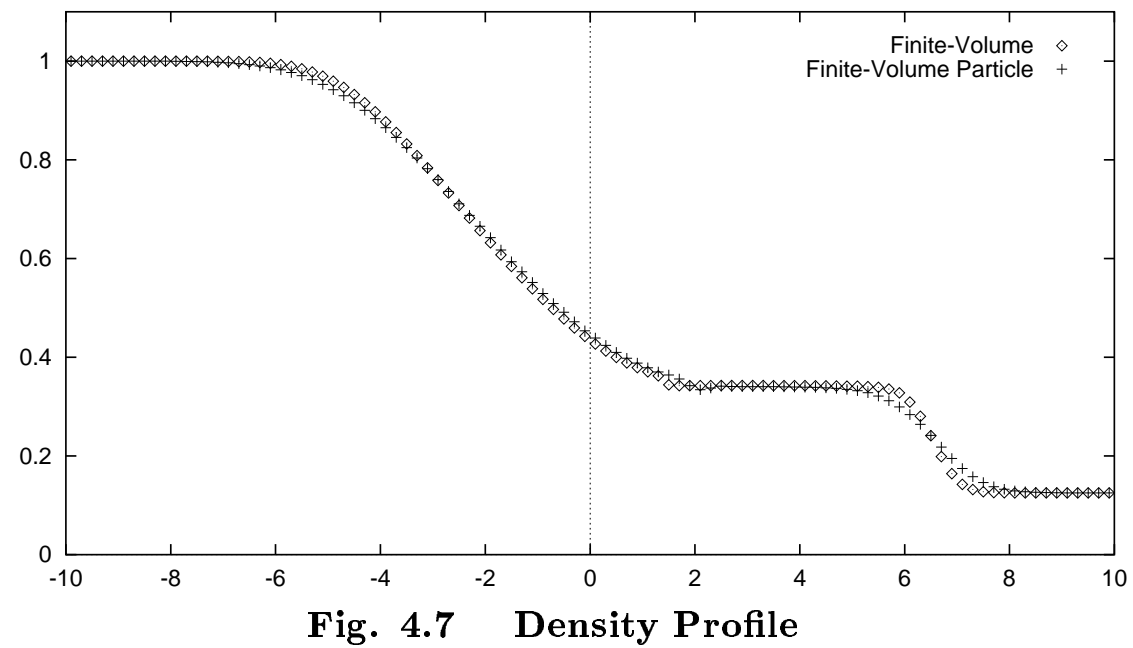

At the transition between the rarefaction wave and the density plateau, both schemes show some irregularities; in particular, the Finite-Volume particle method yields a small velocity overshoot close to the transition point. One reason for this, is that applying the FiniteVolume particle scheme in conservation form, the velocity profile $u(t, x)$ is computed using the interpolation formula

$$
u(t, x)=\frac{1}{\sigma(t, x)} \sum_{i=1}^{n} \frac{\left(V u_{2}\right)_{i}}{\left(V u_{1}\right)_{i}} W\left(x, x_{i}\right)
$$

and one has to use the ratio of the two discrete quantities $\left(V u_{1}\right)_{i}$ and $\left(V u_{2}\right)_{i}$, i.e. small deviations from the "exact" value may yield large deviations in the quotient.

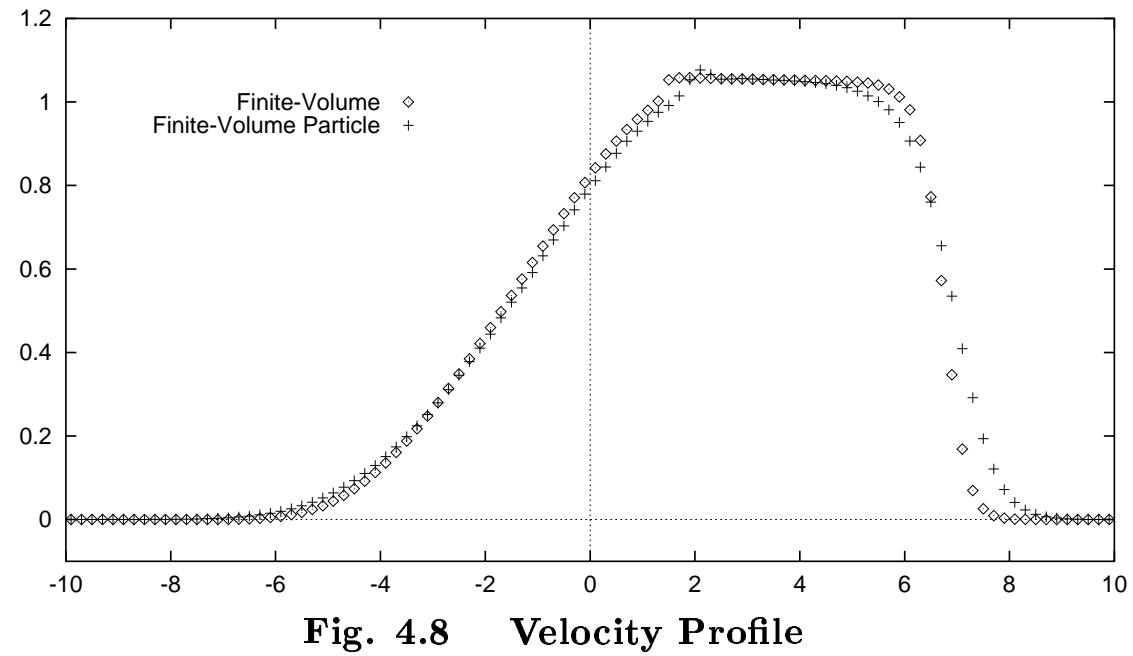

The corresponding discrete particle characteristics together with the spatial positions are shown in Fig. 4.9. First of all, one may observe, that the particle positions are no longer equidistant due to non-vanishing velocity profile: the distance between particles in the rarefaction region increases, whereas the distance decreases along the density plateau. On the other hand, the particle volumes increase in the rarefaction region and therefore, slightly decrease along the density plateau. 


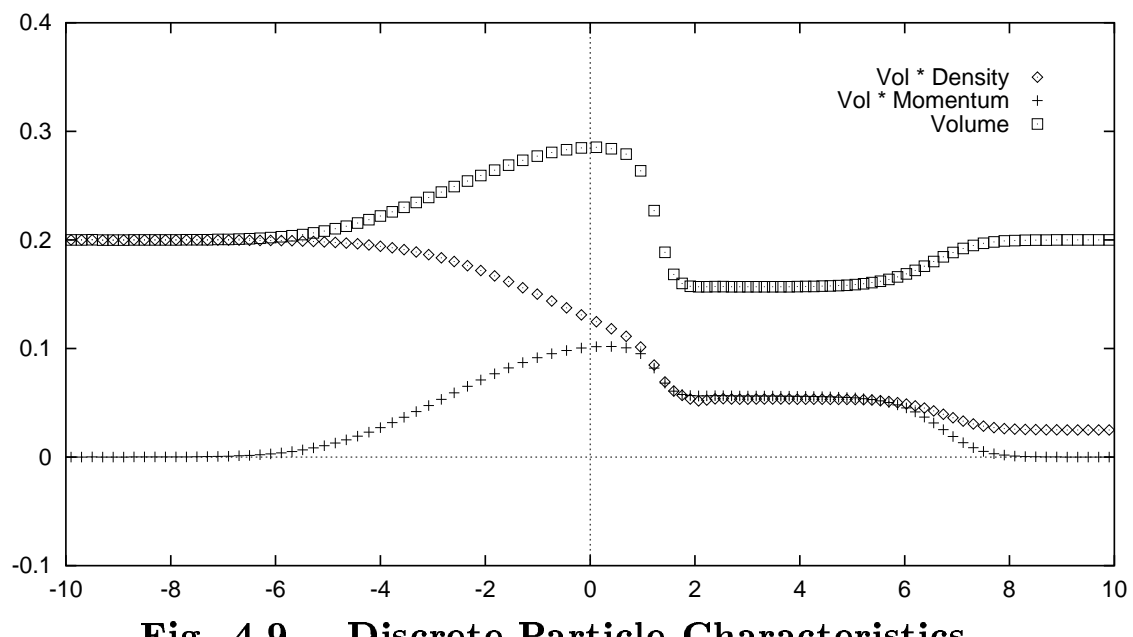

Fig. 4.9 Discrete Particle Characteristics

The irregularity in the velocity profile applying the Finite-Volume particle scheme is caused by a small deviation in the discrete conservative quantities close to the transition between the density plateau and the rarefaction regions and clearly amplified when calculating the ratio of the discrete quantitities.

\section{Conclusion}

One of the drawbacks applying the SPH method for, e.g. the compressible Euler equations, is the concept of the artificial viscosity, which is necessary to resolve shock wave solutions. On the other hand, the method seems to be quite attractive due to its completely grid-free formulation. Using a Finite-Volume scheme, a numerical viscosity is a-priori included via the use of a numerical flux function and this directly stabilizes the scheme when computing shock waves.

In the current investigation we concentrated on the idea to combine the advantages of a particle scheme, i.e. the grid-free formulation, with the concept of a numerical flux function as used in the classical Finite-Volume method. The combination between the two approaches was achieved using a special class of test functions, which are obtained from a generic smoothing kernel localized at the current particle positions. The resulting equations of motions for the discrete particle set contain terms, which include a numerical flux function similar to the standard Finite-Volume scheme, and some correction terms due to moving particles. Hence, a numerical viscosity and therefore a stabilization is a-priori contained and no artificial viscosity term need to be included in the equations of motion.

The new particle method was tested against a classical Finite-Volume scheme for a Riemann problem of the Burgers equation as well as the one-dimensional shock tube problem governed by the isentropic Euler equations and the numerical results were quite promising. On the other hand, a further investigation of the scheme from a theoretical as well as numerical point of view is necessary and currently under investigation. 


\section{References}

[1] R. Dilisio, E. Grenier AND M. Pulvirenti, On the convergence of the SPH method, preprint, to appear in: Computers and Mathematics with Applications

[2] D.A. FulK AND D.W. Quinn, An Analysis of 1-D Smoothed Particle Hydrodynamics Kernels, J. Comp. Phys., 126, 165-180 (1996)

[3] D. KRöneR, Numerical Schemes for Conservation Laws, Wiley \& Teubner, Chichester/Stuttgart, 1997

[4] R. LeVeque, Numerical Methods for Conservation Laws, Birkhäuser, Basel, 1990

[5] J.J. Monaghan, Smoothed Particle Hydrodynamics, Annu. Rev. Astron. Astrophys., 30, 543-574 (1992)

[6] J.P. MoRRIS, P.J. Fox AND Yi ZHU, Modeling Low Reynolds Number Incompressible Flows Using SPH, J. Comp. Phys., 136, 214-226 (1997)

[7] J.P. Morris and J.J. Monaghan, A Switch to Reduce SPH Viscosity, J. Comp. Phys., 136, 41-50 (1997)

[8] K. OelSCHLÄGER, On the connection between Hamiltonian Many Particle Systems and the Hydrodynamical Equations, Arch. Rat. Mech. Anal., 115, 297-310 (1991)

[9] J.L. STEGER AND R.F.WARMING, Flux vector splitting of the inviscid gasdynamic equations with application to finite-difference methods, SIAM J. Numer. Anal., 5, 263-293 (1968)

[10] J. Struckmeier, Particle Methods in Fluid Dynamics, preprint, to appear in the Proc. of the $3^{\text {rd }}$ Conference on "Numerical Modelling in Continuum Mechanics, Prague 1997

[11] J.P. VILA, Convergence of SPH method for scalar nonlinear conservation laws, preprint, University Toulouse, 1995

[12] H. YSEREnTANT, A new class of particle methods, Numer. Math., 76, 87-109 (1997)

[13] H. Yserentant, A particle method for compressible fluids, Numer. Math., 76, 111-142 (1997) 